\title{
Alignment and Calibration of high frequency ultrasound (HFUS) and optical coherence tomography (OCT) 1D transducers using a dual wedge-tri step phantom
}

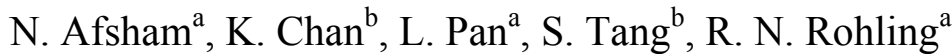 \\ ${ }^{a}$ Robotics and Control Lab., Dept. of ECE, UBC, 2332 Main Mall, BC, CA \\ ${ }^{\mathrm{b}}$ Microsystems and Nanotechnology Group, Dept. of ECE, UBC, 2332 Main Mall, BC, CA
}

\begin{abstract}
This paper introduces a novel alignment and calibration method for high frequency ultrasound (HFUS) and optical coherence tomography (OCT) 1D transducers. 2D images are constructed by means of translation of the transducers using a linear motor stage. Physical alignment of the transducers is needed in order to capture images of the same crosssectional plane, and calibration is needed to determine the relative coordinates of the images, including the image skew. A dual wedge-tri step phantom is created for both alignment and calibration. This phantom includes two symmetrical wedges and three steps that provide the user with visual feedback on how well the scan plane is aligned with the midplane of the phantom. The phantom image consists of five line segments, each of which corresponds to one of the wedges or steps. The slopes and positions of the lines are extracted from the image and compared with the phantom model. The scan plane parameters are found so that the difference between the model and extracted features is minimized. The main advantage of this phantom is that only one frame is required to determine translations, orientations, and skew parameters of the scan plane with respect to the phantom. Experimental results with ocular imaging show the ability to achieve alignment based on this method and its potential for medical applications.
\end{abstract}

Keywords: Calibration, Alignment, Phantom, High Frequency Ultrasound (HFUS), Optical Coherence Tomography (OCT)

\section{INTRODUCTION}

In order to determine the exact position of a feature in a captured image with respect to global coordinate system, calibration of the imaging device is needed. This imaging tool can be a camera or a medical imaging probe. Calibration accuracy is often a critical issue in medical imaging. Inaccurate localization of anatomy in the space can lead to incorrect diagnosis, mislocation of treatment in the case of therapy, or inaccurate navigation in image guided surgery.

In addition to calibration, some medical applications require physical adjustment of the plane to a desired pose in the global coordinate system. This adds an extra step after calibration, which henceforth is called alignment. Although calibration has been studied extensively, little work has focused on the addition of the alignment step. One of the basic applications of alignment is image fusion of two different modalities from the same region of interest. For example high frequency ultrasound (HFUS) measures acoustic reflective properties in intravascular [1], skin and eye [2] domains. Other ultrasound-based imaging techniques, such as photo-acoustic tomography and acousto-optical tomography, strain imaging and elastography, can reveal other properties of tissues distinct from the ultrasound (US) images [3], [4]. Optical Coherence Tomography (OCT) is another fast growing non-invasive and high resolution biomedical imaging modality which has been considered as an optical counterpart of HFUS. Each combination of the aforementioned imaging modalities increases the amount of medical information of the imaging area. The key step is the registration of the various images into a common coordinate system so that measurement from the same location in space can be compared. One approach is to take multiple images from approximately the same region and create a common intermediate slice through interpolation. Unfortunately, the quality of the interpolation is now a function of the interpolation methods and

Medical Imaging 2011: Visualization, Image-Guided Procedures, and Modeling,

edited by Kenneth H. Wong, David R. Holmes III, Proc. of SPIE Vol. 7964, 796428

(c) 2011 SPIE · CCC code: $1605-7422 / 11 / \$ 18 \cdot$ doi: $10.1117 / 12.878182$

Proc. of SPIE Vol. 7964 796428-1 
the density of data, which may be sparse in some applications. The best approach is to precisely align probes prior to imaging. This alignment increases the accuracy, dependability and simplicity of later data fusion as well as interpretation.

Many of the listed modalities are based on moving a 1D transducer to create a 2D image. Alignment is therefore needed for multiple 1D sources to a common direction of motion. In some applications, the 1D source must be aligned to the

same acquisition direction. For example, recent research on enhancement of OCT imaging using US [5], [6] opens a new opportunity to increase the penetration depth of OCT via light-sound interactions. Huang et al. [5] state that misalignment of the US and OCT beams influences the results and makes the analysis of this phenomenon more complicated. Therefore it is beneficial to align the US beam with OCT beam and investigate their interactions more precisely.

The main focus of this work is on the fusion of HFUS and OCT for ocular imaging. Central corneal thickness (CCT) is an example measurement in ocular images. Many studies show CCT is a powerful predictor of the development of glaucoma [7]. Likewise in keratorefractive surgery, surgical success and post-procedure complications are highly dependent on the corneal thickness of the subject [8]. Different imaging modalities, such as OCT and ultrasound, have been tested for CCT measurement and distinguishable discrepancies have reported among them [9], [10]. The most reliable way of CCT measurement is still ultrasonic pachymetry [10] which makes use of a 1D ultrasound transducer. During measurement, care must be taken to apply the probe perpendicularly in the center of the cornea. If the probe is not exactly perpendicular, the CCT measurement is affected and may be a source of error in repeated tests.

In this paper we introduce a new method of A-scan alignment using a dual wedge-tri step phantom. Alignment is performed on two 1D transducers and 2D images are created by means of a stepwise linear movement. The formulation of alignment is first written similar to conventional freehand 3D US calibration. The phantom, calibration and alignment methods are all formulated to facilitate, easy and accurate alignment. To the best of our knowledge, there is no previous work on the alignment of OCT and HFUS 1D transducers. This paper represents the first stage of research on CCT measurement using HFUS-OCT fusion.

\section{MATERIALS AND METHODS}

\subsection{System overview}

HFUS and OCT images are created by using a 1D transducer attached with adjustable optical mounts to a common linear motor stage. The goal of alignment is to locate the 1D HFUS and OCT transducers in positions that results in the same scanning plane made by the lateral movement.

The image acquisition system consists of a $50 \mathrm{MHz}$ 1D HFUS transducer (Episcan 2000I, Longport Inc., Chadds Ford, USA) and a customized Spectral Domain OCT (SD-OCT) 1D transducer with a center wavelength of $845 \mathrm{~nm}$. The phantom is made of aluminum and its base part of is a aluminum cube with dimension of $50 \times 60 \times 70 \mathrm{~mm}$. The dual wedge-tri step part has been machined on top of the cube. A mount for the tissue sample is located in front of the phantom so that tissue scanning can be performed immediately after calibration (Fig. 1). The phantom is immersed in a water tank and its position relative to the tank is fixed during each alignment process. The effect of speed of sound is accommodated by considering the water temperature during the experiments. Correct physical alignment of the two transducers is needed to ensure the same scan plane is imaged by the lateral movement of the transducers. The lateral movement is created by means of a linear motor stage (T-LSR150B, Zaber Technologies Inc., Vancouver, Canada) with equal steps of $30 \mu \mathrm{m}$. When each $2 \mathrm{D}$ image is constructed, the pose of the scan plane with respect to the phantom is determined. Then the difference between the scan plane pose and desired pose (mid-plane of the phantom) is reduced by 
adjusting the screw mounts holding the transducer. This procedure is repeated for the other transducer until both transducers create images of approximately the same plane. The features of the phantom then serve to determine the translations, rotations, and skew parameters for both of the transducers. Since the origins are different for the two transducers, the axial and lateral translations may be different without changing the scan plane. The differences of the calibration parameters of two transducers, including translation in the elevation direction and rotation and skew parameters, have been considered as residual alignment errors. To make the final alignment more accurate, this procedure can be repeated. Figure 1(c) depicts an overview of the system.

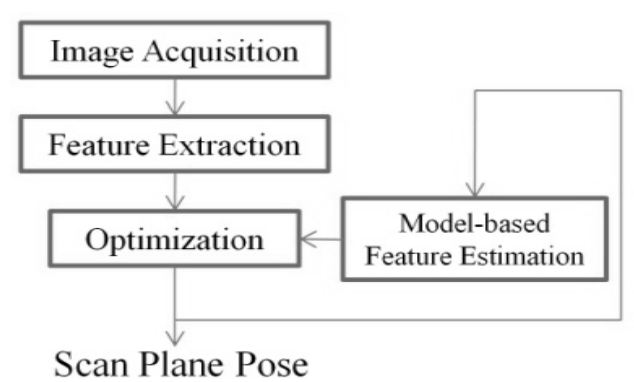

(a)

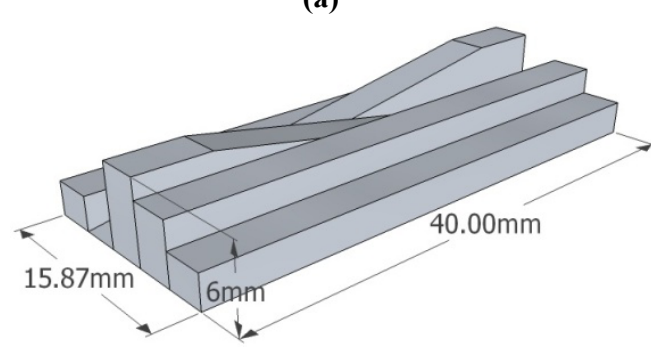

(b)

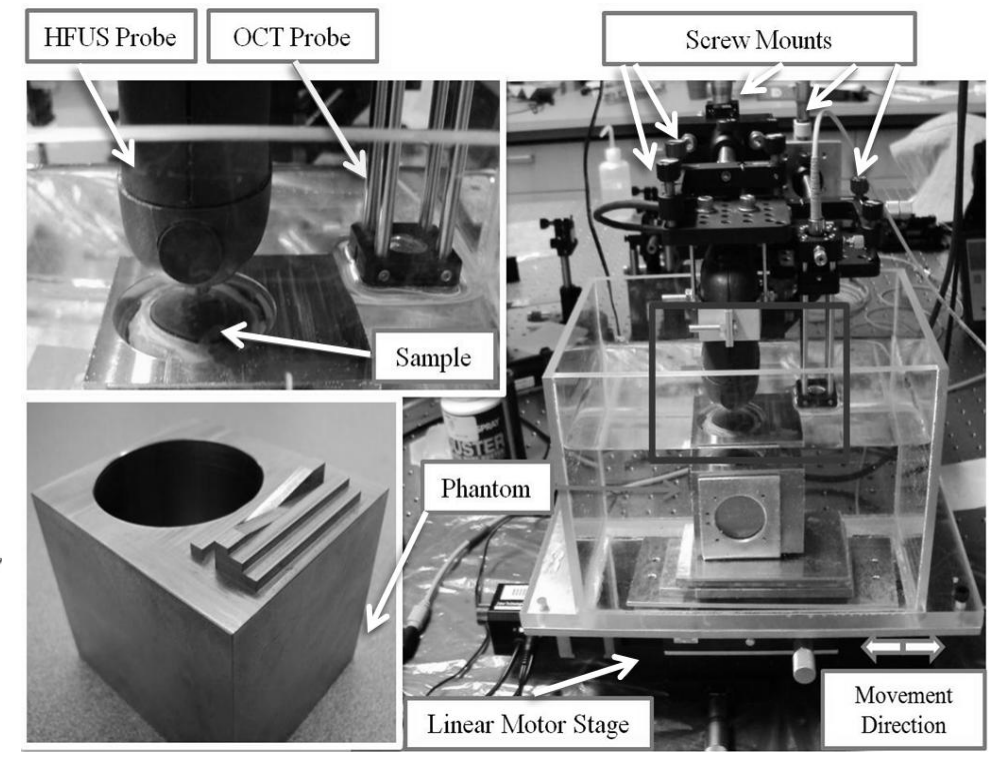

(c)

Figure. 1 Alignment Setup: (a) System block diagram. The parameters of the imaging plane is determined by minimization of the error between the extracted features and the corresponding features from the simulation based on the geometrical model (b) 3D model of the dual wedge-tri step phantom. (c) Both of the transducers are attached with adjustable optical mounts to an optical table. The position of the water tank and phantom are fixed with respect to the linear motor stage during the alignment procedure.

\subsection{Alignment framework}

One approach to alignment is to perform calibration for the US and OCT transducers for a given relative pose, and then physically change their poses to reduce misalignment. Alternating steps of physical alignment and calibration would continue until sufficient alignment is achieved. However it is important to choose a suitable alignment method to reduce the number of steps needed to converge to the desired alignment.

A variety of approaches for calibration of freehand 3D ultrasound have been investigated and they have been compared in terms of reconstruction accuracy, reproducibility, and acquisition time [11]. One of the typical methods of calibration is to image an artificial object, known as phantom, with known geometrical parameters and combine the prior knowledge of the phantom and its ultrasound image to solve for calibration parameters. Theoretically, by knowing the position of three noncollinear points resided in the same ultrasound image and their corresponding location with respect to a position sensor or global frame, the transformation parameters can be determined. However many calibration methods need several images to converge to the solution [12] which make them especially time consuming and inappropriate for the alignment purpose. Some calibration methods need an external tracking system that is also not desired in this application. The main goal of HFUS and OCT 1D transducer alignment is to image the same plane of the sample. As a result, a calibration method which gives the transformation between image coordinate and phantom coordinate is sufficient for this purpose. The well-known Z-wire phantom gives the transformation between image coordinate and phantom in a 
closed form solution with one image [6]; however, visual feedback is not provided on how to adjust the degree of freedoms (DOFs) of one transducer to achieve alignment, so a trial-and-error strategy must be used. This is infeasible given the fact that 5DOF need adjustment for each transducer, and the screw mounts do not provide independent control of the DOF because of non-intersection of the screws' axes. What is needed is a phantom that provides visual feedback for alignment. Wedges are one method for producing such visual feedback. This idea was firstly proposed in [13] where a precision-manufactured mechanical instrument facilitates the alignment of the beam with a plane of wires. It has also been used as an aid for hand-eye coordination based calibration [14]. In this work, the use of wedges has been extended and incorporated into the 1D alignment problem. The goal is to achieve alignment with few iterations through the use of visual feedback of image symmetry. The phantom should also provide a measure of residual misalignment.

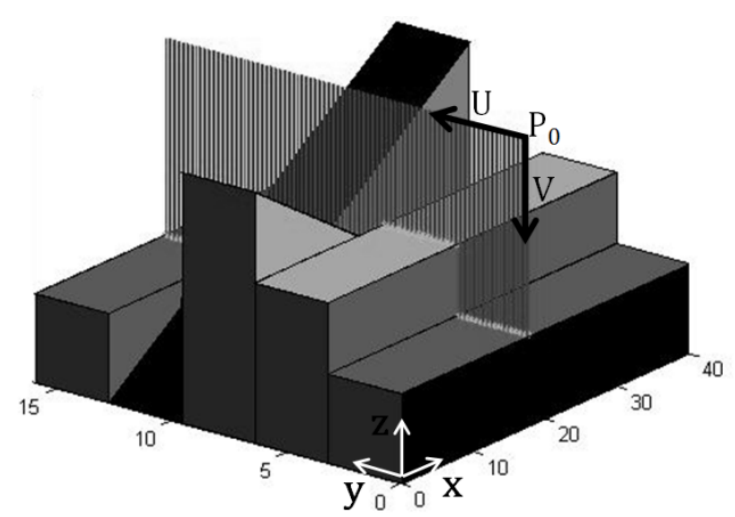

(a) (b)

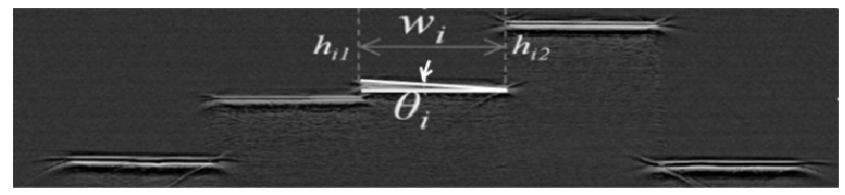

(c)

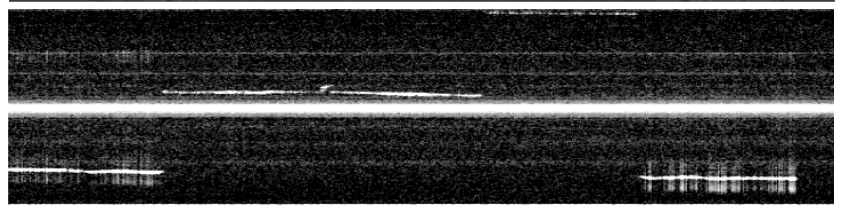

(d)

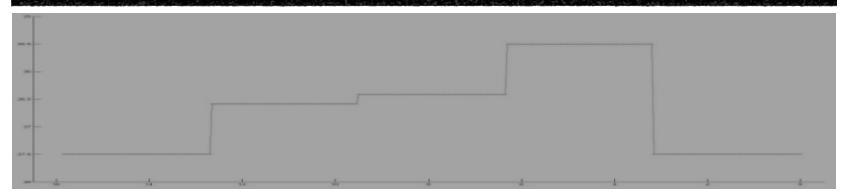

Figure. 2 (a) Simulated phantom and image. $P_{0}$ is the initial position of the probe, $U$ is the trajectory vector along the movement and $V$ is the beam vector. $h_{i 1}, h_{i 2}, w_{i}$ and $\theta_{i}$ are heights, width and angle of the $i$ th segment respectively. (b) HFUS image example of the phantom (c) OCT image example of the phantom (the second segment from right to left is very close to the top of the image). The bright line in the middle of the image is the reference of OCT. To increase the axial range of OCT mirrored signal has been incorporated (d) An example of the model-based segmented line for the final solution of $P_{0}, U$ and $V$.

The proposed approach for computation of the scan plane parameters can be considered as a feature-based registration to a $3 \mathrm{D}$ geometrical model of the phantom that is known to within the manufacturing precision. A simulation of the image construction process is used to create a virtual image for each pose (Fig. 2(a)). Then an iterative nonlinear least squares optimization algorithm is implemented to find the scan plane's pose so that the extracted features from the real image best fit the model-based ones. These features include slopes, heights and widths of the line segments (Fig. 2(b)). Although the angle can be extracted directly from the heights and width of a segment, yet we found that adding the angles to the set of features makes the solution robust and decreases the effect of feature extraction error.

\subsection{Phantom description}

The key aspect of the proposed phantom is that calibration can be done with only one unrestricted scan of the phantom. Figure 1(b) shows the geometry of the phantom. This phantom consists of two symmetrical wedges and three steps. The first and last steps have the same height $( \pm 0.008 \mathrm{~mm})$ and the second step is $2.5 \pm 0.008$ mm higher. The angle of the wedges with $\mathrm{z}$ axis of the phantom is $9.93^{\circ} \pm 0.05^{\circ}$. When imaging such a phantom, a segmented line is created (Fig. 2(b)). Another aspect of the new phantom is that its images provide visually the direction and the amount of misalignment from the desired pose. When the probe beam is nearly perpendicular to the phantom surface, then the changes in the angles of corresponding steps' segments gives information about the movement trajectory. On the other 
hand, when the probe movement trajectory is almost parallel to the x-y plane, the angle of the wedges' segments and the widths of the segments are affected by beam angle. Furthermore the direction of segments' slopes gives roughly the amount of rotational beam misalignment. Lastly, the difference in height of the wedges' segments shows the amount and the direction of the offset along $x$ direction.

In summary, we can adjust the probe to the desired pose by looking at the phantom image, then rotating and translating the probe until all the segments are parallel and the height of the wedges' segments are the same. However adjusting 7 DOF by eye is still a challenge so the heights, widths and angles of each segment are extracted as shown in Figure 2(b) to calculate these 7 DOF accurately.

\subsection{Skew as an additional DOF}

If $P^{I}$ is a point in the ultrasound image, the actual position of the point in the global coordinate $\left(P^{g}\right)$ can be obtained via a homogeneous transformation $\left(T_{I}^{g}\right)$ having six degrees of freedom (DOF) [11]. This general geometric transformation matrix is typically expressed as the multiplication of several transformation matrices

$$
P^{g}=T_{I}^{g} P^{I}=T_{p}^{g} T_{s}^{p} T_{s} P^{I} .
$$

where $T_{s}, T_{s}^{p}, T_{p}^{g}$ are the scaling matrix, the transformation matrix from the image plane to the probe coordinate and the transformation matrix from the probe to the world respectively (Fig. 1 (a)).

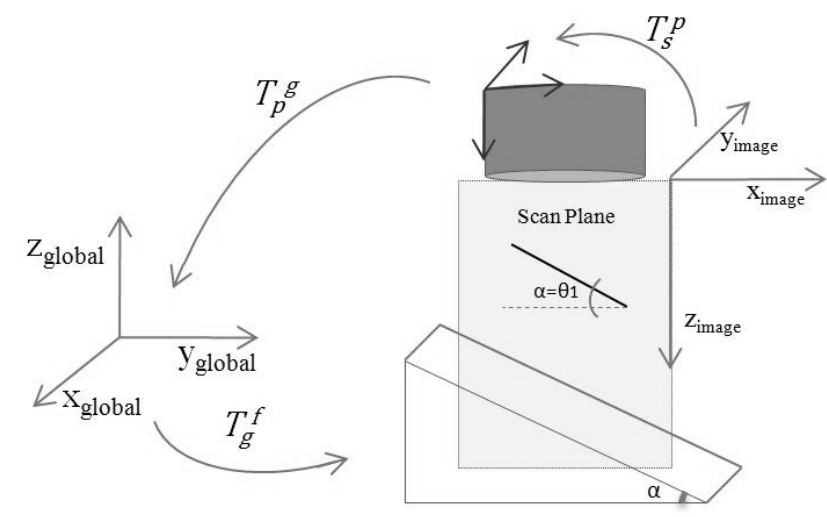

(a)

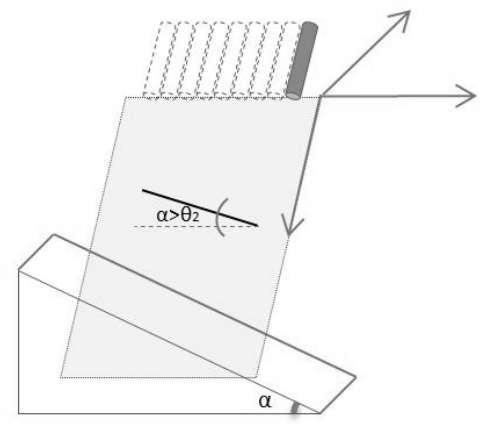

(b)

Figure. 3. (a) A 2D probe in a conventional coordinate system. (b) A moving tilted 1D transducer will cause a wedge with angle of $\alpha$ to be imaged less angled. The dotted shapes show the translated 1D transducer. $T_{s}^{p}, T_{p}^{g}$ and $T_{g}^{f}$ are the transformations from the scan plane to the probe, from the probe to the global frame, and from the global frame to the phantom respectively.

In conventional ultrasound calibration, it is assumed that the axes of the image coordinates are orthogonal, so skew is generally ignored in previous calibration work. However, by constructing an image by translation of a 1D probe, skew is likely significant. The formulation of calibration in non orthogonal image coordinates can be modified by adding a skew parameter to the transformation matrix. In fact we can rewrite $T_{s}^{p}$ as a multiplication of a shear matrix ( $S$ ) followed by rotation $(R)$ and translation $(T)$ matrices. Therefore skew adds another DOF to the 6 previous ones. The effect of skew is delineated in Figure 3(b). 


\section{EXPERIMENTS}

In a series of experiments using the phantom, the HFUS and OCT transducers were aligned to the mid-plane position of the phantom. First, the phantom was imaged from both of the transducers without any change of the tank and phantom positions. Then the previously mentioned features were extracted from both images and nonlinear optimization was performed to solve the pose parameters. Then misalignments from the desired pose were calculated and both of the position and the orientation of the transducers were adjusted by means of several screws based on the calculated misalignments and the visual feedbacks of the images. This procedure was repeated for both of the transducers until the misalignment residual errors could not be improved further. Six independent trials were performed. Each trial consisted of a number of repetitions for both of the transducers. To demonstrate the effect of alignment on imaging, a subsequent experiment was performed on an excised bovine eye using first manual alignment, and then alignment using the proposed method.

\section{RESULTS}

Table 1 summarizes the results of the six independent trials. For each trial, 2 to 6 iterations were needed. Each iteration took 1 to 5 minutes to adjust for the both transducers. The small number of iterations demonstrates the success of the method to achieve alignment with a practical number of iterations and in an acceptable time of performance.

Table 1. Results of alignment errors from desired pose (mid-plane of the phantom) for six independent trials

\begin{tabular}{|l|c|ccccc|}
\hline \multicolumn{2}{|c|}{ Residual errors } & Roll (deg) & Pitch (deg) & Yaw (deg) & Skew (deg) & $\boldsymbol{x}(\boldsymbol{m m})$ \\
\hline \multirow{3}{*}{ HFUS } & Mean & 0.03 & 0.589 & 0.56 & 0.368 & 0.277 \\
& RMS & 0.022 & 0.56 & 0.49 & 0.194 & 0.28 \\
& Max & 0.079 & 1.40 & 0.98 & 0.724 & 0.57 \\
\hline \multirow{3}{*}{ OCT } & Mean & 0.021 & 0.24 & 0.95 & 0.977 & 0.051 \\
& RMS & 0.011 & 0.21 & 0.56 & 0.302 & 0.27 \\
& Max & 0.035 & 0.53 & 1.52 & 1.42 & 0.38 \\
\hline
\end{tabular}

Corneal images with manual and phantom-based alignment are shown in figure 4. It is obvious that when two transducers are aligned the corneal curvature and thickness are more consistent between HFUS and OCT.
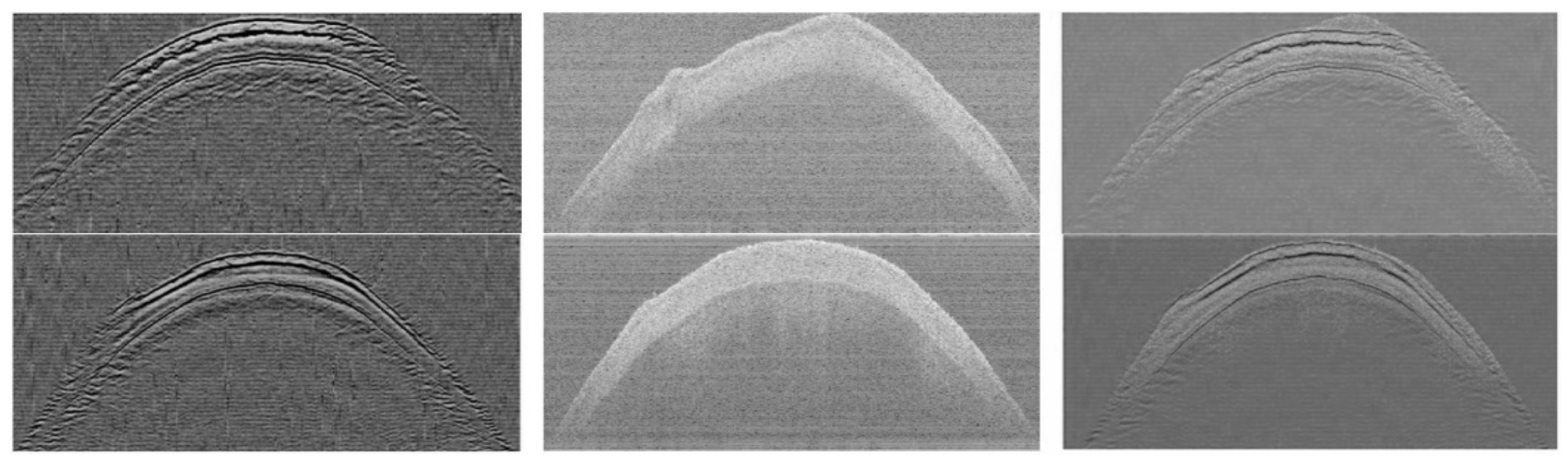

Figure 4. Images from an excised bovine eye with manual and phantom-based alignment.

Left column: HFUS image; middle column: OCT image; right column: Images from the same sample after alignment using the proposed method. Top row: manual alignment, Bottom row: aligned with proposed method. Top row images depict that misaligned probes may result in different CCT measurements. 


\section{CONCLUSIONS}

This paper makes two main contributions. First is a description of the problem of physically aligning two 1D transducers as distinct from calibration. This includes the need for visual feedback in alignment and the incorporation of image skew as an additional DOF. In the proposed method, alignment for two 1D transducers is performed based on scanning of a dual wedge-tri step phantom. For the parameter calculation of the alignment, the angles, the heights and the width of the line segments are extracted from the phantom image and a nonlinear optimization has been performed to solve for these parameters.

The second is the development of a new phantom that provides the capability of visual alignment and computation of calibration parameters from the extracted line features from a single image. Line segments of the phantom image are used to provide an accurate estimate of pose to within sub-millimeter and sub-degree accuracy.

Experimental results demonstrate the ability of this method to align a 1D HFUS transducer and a 1D OCT transducer and to decrease the CCT measurements error by alignment and fusion of OCT and HFUS data. These initial tests suggest areas for the development of a closed-form solution for the calibration step based on the scanning different planes. Further increases in the ease-of-use and accuracy of CCT measurement will be the subject of future research.

\section{ACKNOWLEDGEMENTS}

This work is supported by a grant from the BC-China Innovation and Commercialization Strategic Development Program sponsored by the BC Innovation Council.

\section{REFERENCES}

[1] I.-K. Jang et al., "Visualization of coronary atherosclerotic plaques in patients using optical coherence tomography: comparison with intravascular ultrasound," J Am Coll Cardiol, vol.39, no.4, pp. 604-609, Feb. 2002.

[2] A. Podoleanu, J. Rogers, D. Jackson, and S. Dunne, "Three dimensional OCT images from retina and skin," Optics Express, vol.7, no.9, pp. 292-298, Oct. 2000.

[3] L. V. Wang, "Ultrasound-mediated biophotonic imaging: a review of acousto-optical tomography and photoacoustic tomography," Disease Markers, vol.19, no.2, pp. 123-138, 2004.

[4] K. Parker, M. Doyley, and D. Rubens, "Imaging the elastic properties of tissue: the 20 year perspective," Physics in Medicine and Biology, vol.56, no.1, 2011.

[5] C. Huang, B. Liu, and M. E. Brezinski, "Ultrasound-enhanced optical coherence tomography: improved penetration and resolution," Journal of the Optical Society of America A, vol.25, no.4, pp. 938-946, Apr. 2008.

[6] J. O. Schenk and M. E. Brezinski, "Ultrasound induced improvement in optical coherence tomography (OCT) resolution," Proceedings of the National Academy of Sciences of the United States of America, vol.99, no.15, pp. 9761-9764, Jul. 2002.

[7] L. W. Herndon, J. S. Weizer, and S. S. Stinnett, "Central corneal thickness as a risk factor for advanced glaucoma damage," Archives of Ophthalmology, vol.122, no.1, pp. 17-21, 2004.

[8] I. G. Pallikaris, G. D. Kymionis, and N. I. Astyrakakis, "Corneal ectasia induced by laser in situ keratomileusis," Journal of Cataract and Refractive Surgery, vol.27, no.11, pp. 1796-1802, Nov. 2001.

[9] J. W. McLaren, C. B. Nau, J. C. Erie, and W. M. Bourne, "Corneal thickness measurement by confocal microscopy, ultrasound, and scanning slit methods," American Journal of Ophthalmology, vol.137, no.6, pp. 10111020, Jun. 2004.

[10] D. Y. L. Leung, D. K. T. Lam, B. Y. M. Yeung, and D. S. C. Lam, "Comparison between central corneal thickness measurements by ultrasound pachymetry and optical coherence tomography," Clinical \& Experimental Ophthalmology, vol.34, no.8, pp. 751-754, Nov. 2006.

[11] P.-W. Hsu, R. W. Prager, A. H. Gee, and G. M. Treece, "Freehand 3D Ultrasound Calibration: A Review," in Advanced Imaging in Biology and Medicine, 2009, pp. 47-84.

[12] L. Mercier, T. Langø, F. Lindseth, and L. D. Collins, "A review of calibration techniques for freehand 3-D ultrasound systems," Ultrasound in Medicine \& Biology, vol.31, no.2, pp. 143-165, Feb. 2005. 
[13] A. H. Gee, N. E. Houghton, G. M. Treece, and R. W. Prager, "A mechanical instrument for 3D ultrasound probe calibration," Ultrasound in Medicine \& Biology, vol.31, no.4, pp. 505-518, Apr. 2005.

[14] E. M. Boctor, I. Iordachita, M. A. Choti, G. Hager, and G. Fichtinger, "Bootstrapped ultrasound calibration," Studies in Health Technology and Informatics, vol.119, pp. 61-66, 2006.

[15] A.H. Gee, N.E. Houghton, G.M. Treece and R.W. Prager, "3D ultrasound probe calibration without a position sensor," Technical report CUED/F-INFENG/TR 488, Cambridge University, Department of Engineering, August 2004. 